\title{
Configuração Morfológica do Cone do Amazonas a Partir da Integração de Dados Batimétricos
}

\author{
Luiz Carlos Torres; Izabel King Jeck e Ana Angélica Ligiéro Alberoni Tavares \\ Centro de Hidrografia da Marinha
}

Rua Barão de Jaceguai, S/N Ponta da Armação Niterói RJ 24048-900.

Telefone: 2613 8191. fax: 26138237

E-mail: luiz_torres@hotmail.com

\begin{abstract}
RESUMO
A integração dos resultados encontrados na revisão bibliográfica sobre o cone submarino do Amazonas com a base de dados batimétricos formada por informações coletadas pelo Programa Brasileiro para a Delimitação da Plataforma Continental (LEPLAC) e outras provenientes de domínio público como (GEODAS GEOphysical Data System for Marine Geophysical Data NGDC/NOAA, versão 3), permitiu determinar a extensão e a morfologia do cone.
\end{abstract}

Palavras chaves: cone do Amazonas, banco batimétrico e LEPLAC

\begin{abstract}
The integration of the selected results of previous investigations with bathymetric data sets collected by the Brazilian continental shelf delimitation Program (LEPLAC) and other bathymetric surveys of public domain like (GEODAS GEOphysical Data System for Marine Geophysical Data - NGDC/NOAA, version 3) allowed obtaining the extension and geomorphic delineation of the Amazon submarine fan.
\end{abstract}

Keywords: Amazon fan, bathymetry dataset and EPLAC

\section{Introdução}

O trabalho em questão tem por objetivo apresentar uma abordagem morfológica atualizada da área do Cone do Amazonas com a finalidade de delimitar a referida feição por meio da integração de informações constantes em trabalhos anteriores realizados na área de estudo, nos quais foram empregados métodos de pesquisa diversos, com as informações batimétricas contidas em um banco digital composto por dados coletados durante as comissões LEPLAC (Programa de Levantamento da Plataforma Continental Brasileira), dados do Projeto REMAC (Reconhecimento da Margem Continental Brasileira), dados do Projeto EQUANT (Cooperação Internacional entre a Petrobrás e a Universidade do Estado de Origem - U.S.A.) e dados oriundos do banco de dados GEODAS (GEOphysical Data System for Marine Geophysical Data - NGDC/NOAA, versão 3).

\section{Caracterização da Área de Estudo}

O cone do Amazonas é uma das feições mais proeminentes da margem continental brasileira. Sua origem é descrita por vários pesquisadores (Damuth \& Kumar, 1975; e Debrant et. al., 1997), como conseqüência do soerguimento da cordilheira dos Andes durante o Mioceno médio, quando a drenagem do Amazonas teve seu sentido invertido do oceano Pacífico para o Atlântico. Em paralelo com a inversão, verificou-se uma oscilação para menos no nível médio do mar da ordem de $100 \mathrm{~m}$, possibilitando que grandes quantidades de sedimentos se depositassem além da plataforma continental.

A formação do cone pode ser atribuída, além das flutuações do nível do mar, à bifurcação de canais e movimentos de massa de elevadas viscosidade e densidade (Damuth \& Kumar, 1975). O embasamento sob o cone do Amazonas é de difícil interpretação devido à grande espessura de sedimentos. A margem continental na região do cone apresenta uma morfologia bastante peculiar. A plataforma dos estados do 
Pará e Amapá estende-se por aproximadamente 300 $\mathrm{km}$ a partir da foz do rio Amazonas exibindo cobertura sedimentar arenosa entrecortada por numerosos canais e apresentando a sua quebra em profundidades que variam entre $90 \mathrm{~m}$ e $120 \mathrm{~m}$. O talude e elevação continentais são alterados pelo aporte sedimentar anômalo, que atinge centenas de quilômetros de distância da costa.

Com base em características morfológicas e acústicas, o cone do Amazonas pode ser dividido em três partes. O cone superior estende-se desde a quebra da plataforma até profundidades de $3.000 \mathrm{~m}$. O canion submarino do Amazonas é a feição mais proeminente desta parte do cone, apresentando relevo relativo da ordem de $500 \mathrm{~m}$ (Damuth \& Kumar, 1975) desde a plataforma até a profundidade de $1500 \mathrm{~m}$, onde dá lugar a dois canais distributários com $200 \mathrm{~m}$ de relevo relativo (Damuth et. al., 1988). O cone médio estendese da faixa de profundidades de $3.800 \mathrm{~m}$ a $4.200 \mathrm{~m}$. Caracteriza-se pela presença de inúmeros canais distributários flanqueados por diques marginais "levees" que se superpõem e coalescem lateralmente. Nesta porção do cone os processos erosionais típicos de ocorrerem em talude continental não são abundantes como na porção superior. Pode-se observar, contudo algumas feições erosivas resultantes da turbulência de correntes de turbidez. O cone inferior estende-se até a profundidade de $4.800 \mathrm{~m}$ onde se junta com a planície abissal de Demerara, apresentando reduzido gradiente. Nesta região os canais apresentam-se bastante suavizados ocorrendo extensas áreas aplainadas pelos depósitos distais da sedimentação turbidítica.

A espessura sedimentar média do cone varia de $18.000 \mathrm{~m}$ (superior/médio) a $1.000 \mathrm{~m}$ no cone inferior (Damuth, 1975). As correntes de turbidez e os fluxos de sedimentos transportam sedimentos terrígenos sobre o cone do Amazonas via canais distributários. Quantidade apreciável de sedimentos finos ultrapassaram o limite do cone inferior, sendo depositados na planície abissal. Atualmente, o cone é recoberto por uma fina camada de vasa pelágica marrom, que acompanha a morfologia e denota a ausência de sedimentação terrígena desde o término da última glaciação.

\section{Dados Utilizados}

Para o desenvolvimento deste trabalho foram utilizados dados batimétricos adquiridos pelas comissões LEPLAC-DHNDELTA (DHN, 1997), LEPLAC-VI e LEPLAC-VIIIA. Estes dados foram adquiridos com ecobatímetro Atlas-Krupp e posicionados por GPS. Por seguir uma metodologia uniforme ao longo de todo o seu processo de coleta e ainda por adotar parâmetros de aquisição segundo critérios estabelecidos pela Organização Hidrográfica Internacional (IHO), os dados do LEPLAC podem ser considerados de alta confiança. Perfazem cerca de $12.000 \mathrm{~km}$ de dados. Os dados batimétricos oriundos do GEODAS foram adquiridos em cruzeiros geofísicos de aproximadamente 120 comissões patrocinadas por diversas instituições de pesquisa, em épocas distintas, com métodos e objetivos diferentes, o que implica na necessidade de cuidados especiais na sua utilização, uma vez que a sua qualidade não pode ser assegurada.

A pesquisa bibliográfica proporcionou a base para a interpretação dos dados. A figura 1 apresenta a disposição dos dados batimétricos utilizados para a carga da base de dados empregada para a confecção dos mapas constantes deste trabalho.

\section{Metodologia}

O primeiro passo foi uma pesquisa bibliográfica em estudos publicados sobre a região em questão. Foi constatada uma tendência em delimitar o cone de uma forma semelhante por todos os autores consultados. Como cada trabalho foi fruto do emprego de métodos de investigação diversos, esta heterogeneidade na metodologia empregada era de certa forma esperada. $\mathrm{O}$ conhecimento das cotas batimétricas é um recurso fundamental para a caracterização da morfologia do leito submarino. Assim, foi desenvolvido um banco de dados batimétricos englobando as informações batimétricas disponíveis em formato digital. Devido à heterogeneidade característica dos dados oriundos do GEODAS, principalmente no que concerne seus métodos de aquisição e a sua conseqüente precisão associada, tais informações foram preliminarmente submetidas a um processo de análise e depuração por meio de cruzamento de linhas. Quando possível, esta comparação foi efetuada com os dados do LEPLAC, os quais foram utilizados como padrão para a validação do banco de dados batimétricos. Dessa forma foi possível se fazer uma avaliação dos dados obtidos no GEODAS e estabelecer um controle de qualidade geral para a batimetria inserida no banco de dados. Nos pontos onde ocorreram discrepâncias, as profundidades do banco de dados GEODAS foram linearmente ajustadas às profundidades coletadas no LEPLAC ou desprezadas. No caso em que os dados GEODAS apresentassem problemas, quando a recuperação não era possível, toda a linha foi descartada.

Devido à grande extensão da área de estudo, os dados disponíveis não apresentavam uma padronização de distribuição espacial, e dessa forma, verificou-se uma concentração de informações na porção "oeste" da área em relação à menor concentração verificada no setor "leste". Essa limitação apresentada pelo banco não chegou a comprometer os resultados alcançados. O banco de dados batimétrico totalizou aproximadamente 342.000 informações batimétricas, as quais foram então apresentadas sob a forma de mapas batimétricos de gradiente e de contorno, com o intuito de, juntamente com as provenientes da pesquisa bibliográfica, contribuir para a apresentação da geomorfologia do cone do Amazonas.

Para a confecção dos referidos mapas foi empregado um software de edição de dados e apresentação em mapas (Geosoft, 1996). Após terem sido devidamente validados os dados foram admitidos para confecção da grade batimétrica, segundo o método da curvatura mínima e espaçamento de celas de 5000m. Para a confecção das linhas de contorno, o 


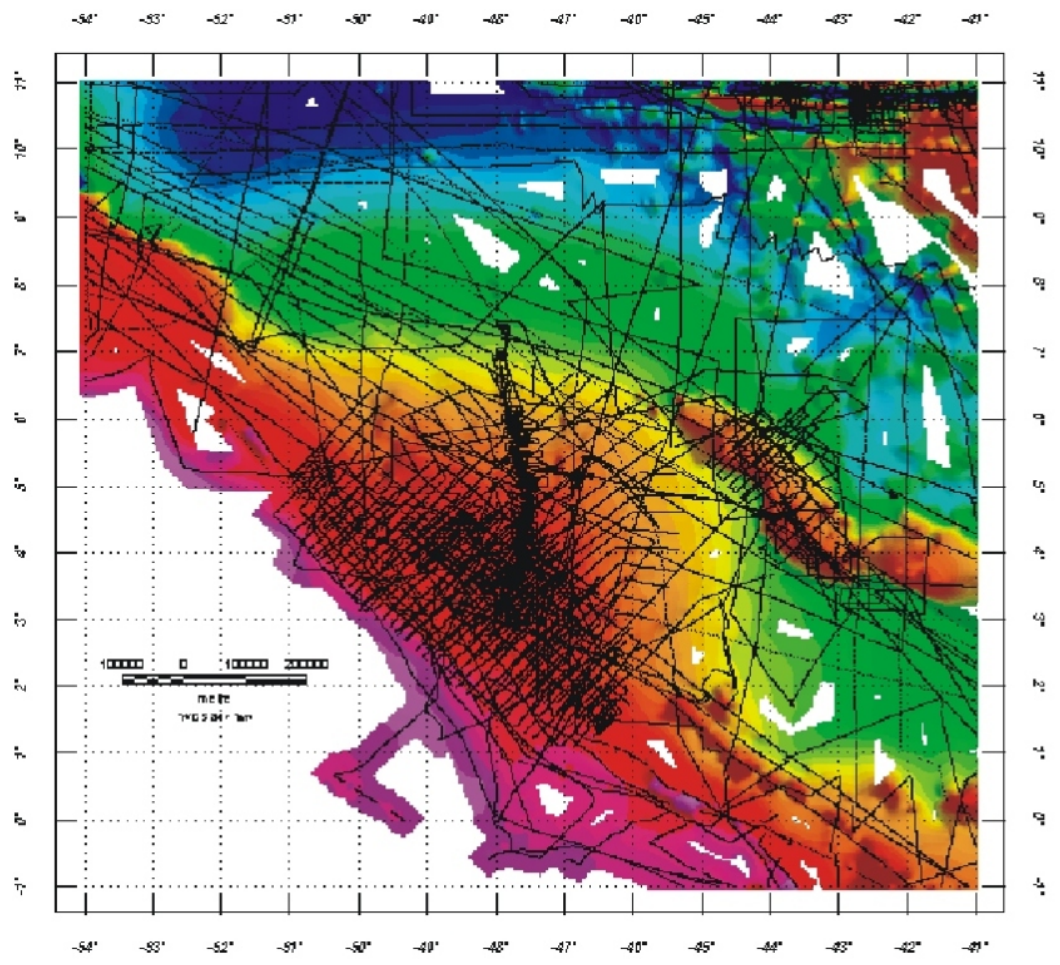

Figura 1 - Mapa contendo a disposição das linhas batimétricas provenientes do GEODAS e do LEPLAC, usadas para carregar a base de dados e a grade batimétrica correspondente. Os pontos em branco correspondem às áreas desprovidas de dados ou em quantidade insuficiente.

software possui uma rotina aonde só necessita ser informada qual a primeira isóbata e qual a diferença para a próxima a ser traçada a partir da grade batimétrica. Para a confecção da grade de inclinação, calculou-se a inclinação entre pontos consecutivos nas direções " $x$ " e " $y$ " e a direção total da inclinação do fundo marinho foi obtida pela soma vetorial das direções individuais.

\section{Discussão dos Resultados}

A partir do banco de dados batimétricos foram produzidos dois mapas: mapa de variação total da inclinação do fundo do mar (gradiente) e o mapa de contorno batimétrico (composto por isóbatas afastadas de $50 \mathrm{~m}$ ), mostrados nas figuras 2 e 3 , respectivamente. As informações retiradas de ambos os mapas se complementam e assim pode-se ter uma melhor idéia da geomorfologia da feição estudada. A desenvolvida plataforma da região, adjacente à foz do rio Amazonas encontra-se evidenciada na Figura 3 entre as isóbatas de $50 \mathrm{~m}$ e $100 \mathrm{~m}$. O grande espaçamento verificado entre essas duas linhas também se manifesta na Figura 2, atribuindo para essa área um baixo gradiente.

A divisão do cone segundo características geomorfológicas e acústicas (Damuth \& Kumar, 1975; Damuth et. al., 1983; Manley \& Flood, 1988; Damuth et al., 1988 e Araújo, 1994), foram retratadas em ambos os mapas. O mapa de contorno reproduziu com considerável resolução a geomorfologia da área retratando os canais distributários e as feições de deslocamento de massa ocorridos no cone superior. O mapa de gradiente endossa essa geomorfologia, quantificando nessa região (quebra da plataforma até a profundidade de $3.000 \mathrm{~m}$ ) as maiores declividades observadas em toda a feição (Torres et. al., 1999).

No cone médio os registros de processos próprios de ocorrerem em região de talude ainda são observados, porém com menor freqüência que na parte superior. Isso já era de se esperar, pois as isóbatas apresentam um razoável intervalo e a inclinação do fundo é mais suave, traduzindo um ambiente, relativamente, de menor instabilidade que o anterior. O cone médio apresenta seu limite externo nas proximidades da isóbata de $4.200 \mathrm{~m}$, estando o limite do cone do Amazonas definido pela região denominada cone inferior. Esse limite distal varia de $4.700 \mathrm{~m}$ (Manley \& Flood, 1988 e Damuth et. al., 1988), passando por $4.800 \mathrm{~m}$ (Damuth et. al., 1983) até $4.850 \mathrm{~m}$ (Damuth \& Kumar, 1975). Para efeito desse trabalho, adotou-se a isóbata de $4.850 \mathrm{~m}$ como limite do cone. Esse limite atinge localmente as regiões de inclinação 1:1.000, alcançando a planície abissal de Demerara.

Os limites laterais do cone não puderam ser definidos por uma única isóbata. Na porção nordeste o limite é condicionado pela barreira estrutural representada pela elevação do Ceará. Na região noroeste e na sudeste, procurou-se demarcar a região com gradientes variando de 1:300 a 1:1.000 (Damuth \& Kumar, 1975). Essas considerações foram incorporadas no mapa batimétrico apresentado na figura 3 apresentando a delimitação do cone do Amazonas. 


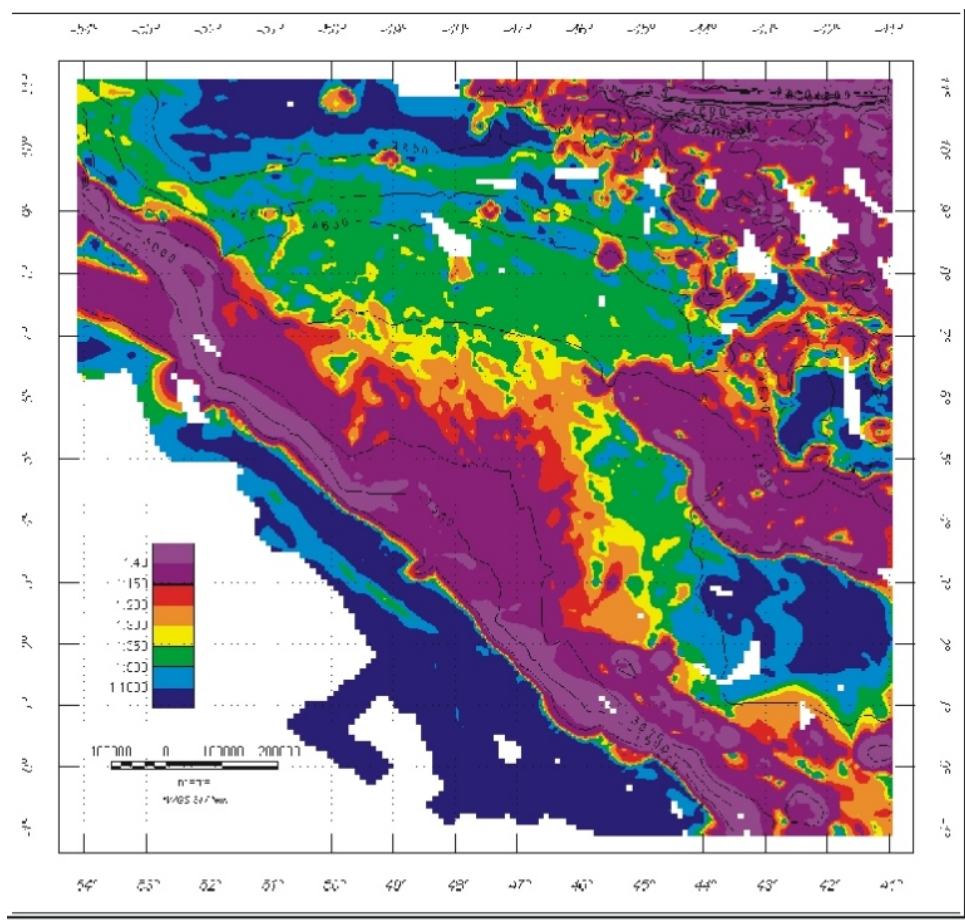

Figura 2 - Mapa de distribuição da inclinação total do fundo oceânico.

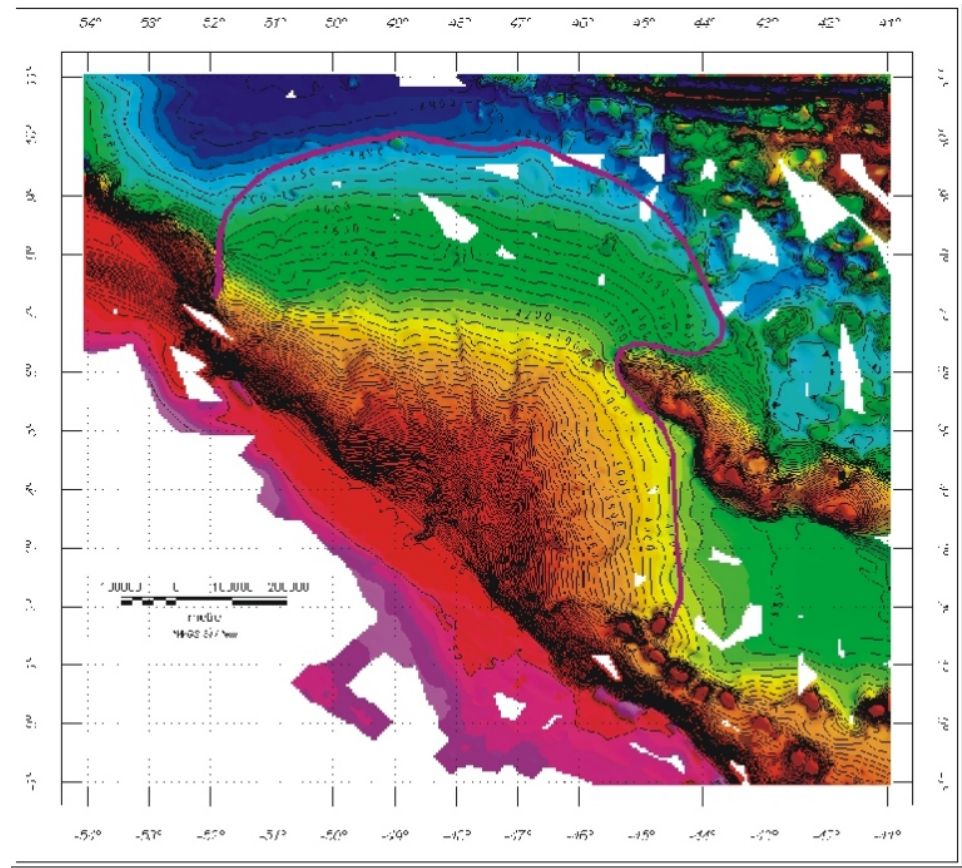

Figura 3 - Mapa batimétrico com linhas de contorno espaçadas de 50m. A Linha encarnada grossa representa o limite proposto para a feição cone submarino do Amazonas. 


\section{Conclusões}

À luz dos dados disponíveis, a aplicação da metodologia adotada possibilitou obter um produto que congrega tanto elementos de ordem quantitativa (isóbatas e regiões de mesma inclinação) quanto de ordem qualitativa (processos erosionais e feições associadas). Os dados batimétricos analisados propiciaram a integração do conhecimento da geomorfologia na região contribuindo para a delimitação do cone submarino do Amazonas. Foi também possível, observar uma sintonia entre os resultados obtidos

neste trabalho com as interpretações propostas por diversos autores consultados, no que se refere à feição em estudo.

Os resultados apresentados neste trabalho, embora não possam ser considerados completos, constituem uma valiosa contribuição para o conhecimento da proeminente feição traduzida pelo cone submarino do Amazonas, principalmente quanto ao caráter morfológico.

\section{Referências Bibliográficas}

Araujo. T.C.M. (1994). Seismostratigraphic Interpretation of the Amazon Continental Margin in View of Sea Level Changes and Subsidence of the Sea Floor, Tese de Doutorado, Kiel Alemanha, 122 páginas.

Damuth. J.E. \& KUMAR. M. (1975). Amazon Cone: Morphology, Sediments, Age and Growth Pattern. In: Geological Society of America, Bulletin V 86, p. 863-878.

Damuth. J. E., KOLLA. V., FLOOD. R.D., KOWSMANN. R, MONTEIRO. M.C., GORINI. M.A., PALMA. J. J.C. \& BELDERSON. R. H. (1983). Distributary Channel Meandering and Bifurcation Patterns on The Amazon Deep-Sea Fan as Revealed By
Long-Range Side-Scan Sonar (GLORIA). In: Geology, V 11, p. 94-98.

Damuth. J.E., FLOOD. R..D., KOWSMANN. R., BELDERSON. R.H. \& GORINI, M.A. (1988). Anatomy and Growth Pattern of Amazon deepsea Fan as Revealed by Long-Range Side Scan Sonar (GLORIA) and High Resolution Seismic Studies. In: The American Association of Petroleum Geologists Bulletin, V 72, N 8, p. 885-911.

Debrant.P., LOPEZ. M. \& CHAMLEY. H.(1997). Clay mineral destruction and significance in Quaternary sediments of the Amazon Fan. In: Flood, R.D., Piper, D.J.K., Klaus, A., \& Peterson, L.C (Eds.), Proceedings of Ocean Drilling Program, Scientific Results, Vol. 155.

DHN - Diretoria de Hidrografia e Navegação, Ministério da Marinha. (1997). Relatório Final do Processamento e Interpretação dos dados batimétricos para a determinação do Pé do Talude Continental e da Isóbata de 2500 metros na Margem Continental Norte Brasileira Cone do Amazonas. Relatório Interno, 101 páginas.

Geodas - GEOphysical Data System for Marine Geophysical Data - NGDC/NOAA, versão 3). Coletânea de CD-ROM, 1995.

Geosoft. (1996). Oasis Montaj data Processing System for Earth Science Applications. Version 4.0. User Guide. Cnadá, 1996, 186p.

Manley. P.L. \& FLOOD. R.D. (1988). Cyclic Sediment Deposition within Amazon DeepSea Fan. In: The American Association of Petroleum Geologists Bulletin, V 72, N 8, p. 912-925.

Torres. L.C., JECK. I.K. \& TAVARES. A.A.L.B (1999) . Integração de dados Batimétricos no Cone do Amazonas. VI Congresso Internacional da Sociedade Brasileira de Geofísica. Rio de Janeiro. SBGf3499-CD 